\title{
Acute-Onset Stuttering as a Lateralizing Neurologic Sign?
}

\section{Jesus J1*, Carvalho $\mathrm{A}^{2}$, Ribeiro $\mathbf{M}^{3}$, Veloso $\mathbf{M}^{2,4}$ and Barros $\mathbf{P}^{2,4}$}

10phtalmology Department, Centro Hospitalar Entre o Douro e Vouga, Portugal

${ }^{2}$ Neurology Department, Centro Hospitalar Vila Nova de Gaia/Espinho, Vila Nova de Gaia,

Portugal

3Imagiology Department, Neuroradiology Unit, Centro Hospitalar Vila Nova de Gaia/Espinho, Vila Nova de Gaia, Portugal

${ }^{4}$ Stroke Unit, Centro Hospitalar Vila Nova de Gaia/Espinho, Vila Nova de Gaia, Portugal

\section{Case Report}

Volume 2 Issue 1

Received Date: May 02, 2018

Published Date: May 24, 2018

DOI: $10.23880 / \mathrm{mhrij}-16000119$

*Corresponding author: Jeniffer Jesus, Ophtalmology Department, Centro Hospitalar Entre o Douro e Vouga, Portugal, Email: j.dominguesjesus@gmail.com

\section{Abstract}

Introduction: Stuttering has been defined as speech dysfluency characterized by involuntary repetitions and prolongations in syllables and words sounds. Acquired stuttering can have a neurogenic etiology, usually following dominant hemisphere stroke.

Case report: A 55-year-old right-handed woman, with history of multiple vascular risk factors and target-organ damage bilateral atherosclerotic carotid disease, symptomatic on the right, for which she was submitted to endarterectomy six years before and three-vessel coronary artery disease treated by percutaneous coronary intervention three years earlier - recurred to emergency department for acute-onset speech disorder at wake-up. On admission she presented stuttering, with preserved naming/repetition/comprehension, and a previously known left claw hand, possibly secondary to posttraumatic ulnar neuropathy (NIHSS 1). Cranial computed tomography (CT) showed non-recent ischemia in corticosubcortical right fronto-parietal region, without acute ischemic signs; angio-CT displayed occlusion of left common carotid artery with patency of ipsilateral internal carotid artery (ICA) and right ICA stenosis $>80 \%$. She received an antiplatelet loading dose and was admitted to our stroke unit. Brain magnetic resonance imaging revealed the non-recent infarction in the right anterior/middle cerebral artery watershed area, surrounded by foci of acute ischemia with restriction to water diffusion. A symptomatic right ICA re-stenosis was assumed; she started double antiplatelet therapy and was submitted to carotid angioplasty with stenting.

Conclusion: Acquired stuttering is more often reported after dominant hemisphere and subcortical lesions, rather than in cortical speech and motor regions. However, this case shows that acquired stuttering may result from non-dominant cortical infarction. Thus, we cannot consider stuttering a focal neither lateralizing neurological sign.

Keywords: Stuttering; Hemisphere; Common Carotid Artery; Computed Tomography 


\section{Mental Health \& Human Resilience International Journal}

\section{Abbreviations}

CCA: Common Carotid Artery; CT: Computed Tomography; ICA: Internal Carotid Artery; MRI: Magnetic Resonance Imaging; NIHSS: National Institutes of Health Stroke Scale.

\section{Introduction}

Stuttering is a speech disorder characterized by disruption of fluent speech, with involuntary repetitions and prolongations in syllables and words sounds [1]. The mechanisms under lying this phenomenon are poorly understood, but two broad types are recognized: primary stuttering, also called persistent developmental stuttering, which often appears in children, without an apparent neurological injury, and occasionally persists in adulthood; and secondary/acquired/neurogenic stuttering, that is less common and usually occurs following a brain injury (e.g. stroke, tumor, head trauma, neurodegenerative diseases as Parkinson's disease...) or drug exposure (e.g. tricyclic antidepressants, phenothiazines). Most reported cases of acquired stuttering secondary to stroke are associated with lesions in the dominant or both hemispheres, and usually, but not always, aphasia is present as well $[2,3]$.

The purpose of this paper is to report the case of a 55year-old right-handed woman who developed acute stuttering, due to a non-dominant cortical infarction.

\section{Case Report}

A 55-year-old right-handed woman with history of several vascular risk factors (dyslipidemia, hypertension and current smoking) complicated by three-vessel coronary artery disease previously treated by percutaneous coronary intervention and by a prior stroke secondary to right symptomatic carotid atherosclerotic disease submitted to endarterectomy six years earlier, under simple anti-platelet therapy, was admitted to the emergency department for an acute-on set speech disorder, detected on awakening. On examination, she showed a disruption of verbal fluency characterized by repetitions, prolongations and blocks of sounds, not restricted to initial syllables, and not associated with secondary motor symptoms such as facial grimacing or eye blinking; her speech was grammatically correct, with preserved naming, repetition and comprehension. Furthermore, she exhibited a previously known left claw hand, possibly secondary to post-traumatic ulnar neuropathy (National Institutes of Health Stroke Scale, NIHSS score of 1 ). The medical history revealed no language problems during childhood, no prior episodes of stuttering and no recent onset of medication.

Cranial computed tomography (CT) showed non-recent ischemia in cortico-subcortical right fronto-parietal topography, without any acute ischemic signs. Angio-CT demonstrated occlusion of the left common carotid artery (CCA) with patency of ipsilateral internal carotid artery (ICA), and right ICA stenosis $>80 \%$, without intracranial large-vessel occlusion (Figure 1).

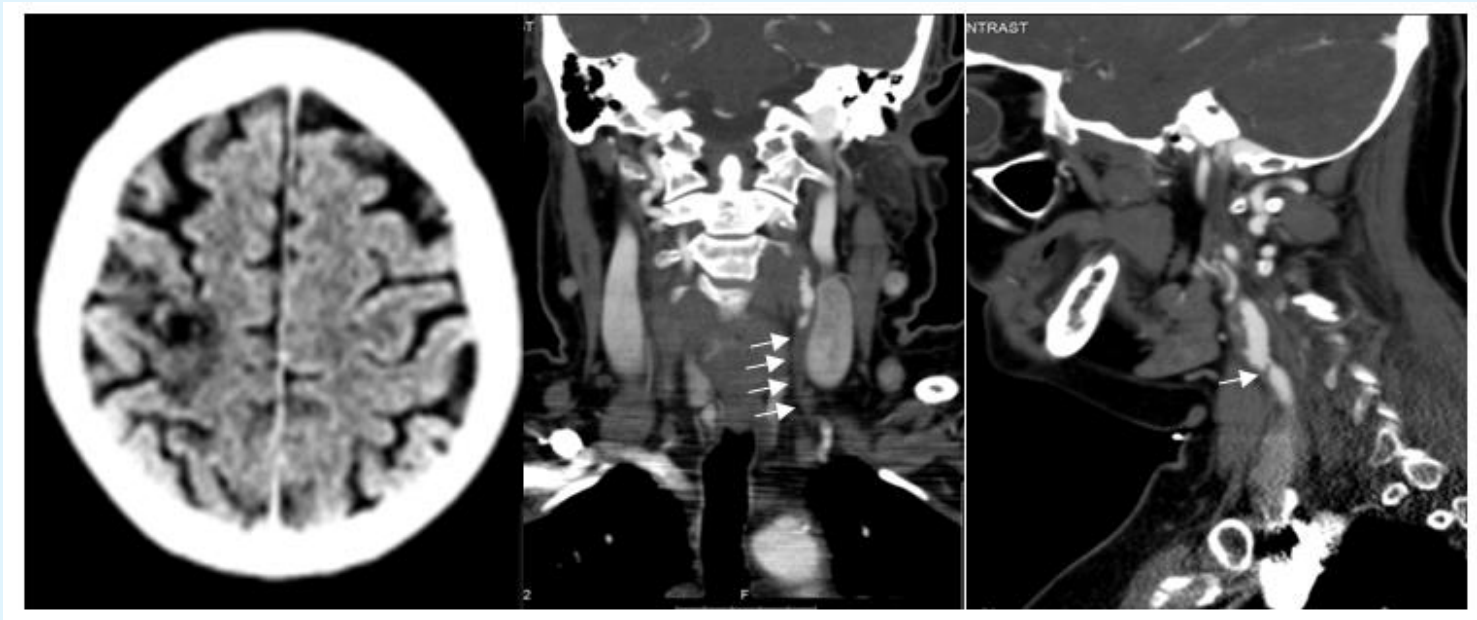

Figure 1: Left image: Cranial CT on admission showing the non-recent ischemia in cortico-subcortical right frontoparietalregion. Angio CT on admission demonstrating occlusion of the left common carotid artery (middle image) and right internal carotid artery stenosis $>80 \%$ (right image). 


\section{Mental Health \& Human Resilience International Journal}

Considering that most cases of stuttering previously reported are due to dominant hemisphere stroke [1,2], we put the hypothesis of symptomatic left CCA occlusion and symptoms resultant from a hemodynamic mechanism, although she showed no response to volume expansion. Though rarer, we could not exclude symptoms secondary to right ICA stenosis. Since it was a wake-up stroke and the patient presented minor neurological deficits, she was not submitted to intravenous thrombolysis; double antiplatelet therapy with a loading dose and highintensity statin were started, associated to permissive hypertension and she was admitted to our stroke unit.

To understand which the symptomatic ICA was, define infarct location and etiologic mechanism, a brain magnetic resonance imaging (MRI) was performed and revealed the non-recent infarction in a watershed distribution between the right anterior and middle cerebralarteries, surrounded by foci with restriction to water diffusion in right fronto-parietal cortex, denoting acute ischemia in right intracranial ICA territory Figure 2. A symptomatic right ICA re-stenosis was assumed. The patient was submitted to right carotid angioplasty with stenting six days after admission. During her stay at the stroke unit, the patient began to improve from the dysfluency. Her pattern of speech started to be slightly more fluent, despite she has continued to prolong and repeat few sounds. Patient was discharged after nine days with high-intensity statin and double anti-platelet therapy during 30 days, and after this period with single antiplatelet lifelong.
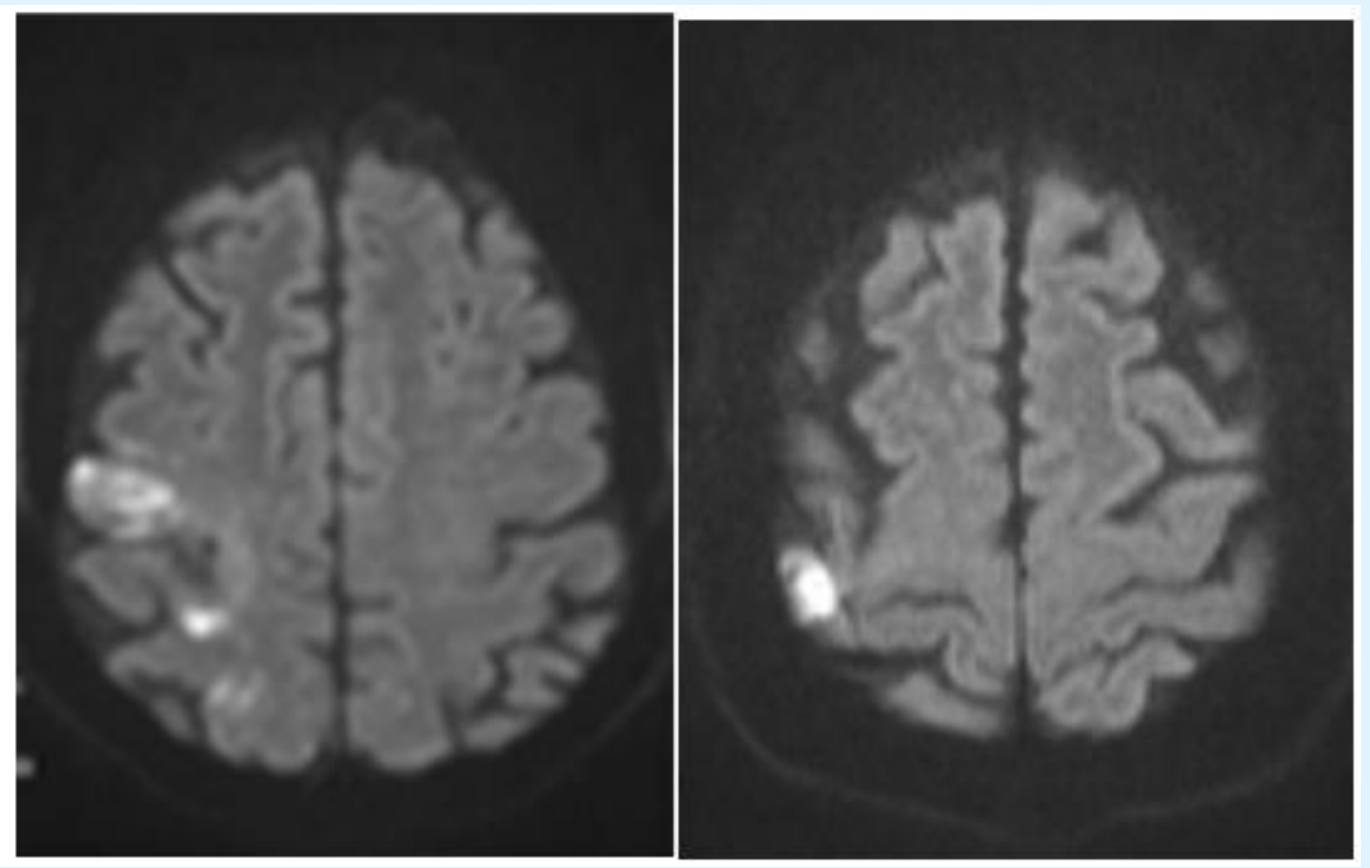

Figure 2: Brain MRI - Diffusion-weighted imaging (DWI) showing right acute fronto-parietal cortical infarction, surrounding the prior ischemic region.

\section{Discussion and Conclusions}

Helm, et al. [4] originally defined the main characteristics of acquired stuttering and described it as a result from either left-sided or bilateral lesions. Although, acquired stuttering is more often reported after dominant hemisphere and subcortical lesions, rather than in cortical speech and motor regions, there are few case reports showing that it can arise from damage on non-dominant hemisphere [1,3,5-7].

The present case involved a patient who developed acuteonset stuttering after cortical infarction in non-dominant fronto-parietal topography, a rarity since most of the described cases have subcortical involvement $[3,6,7]$. 


\section{Mental Health \& Human Resilience International Journal}

Despite more than 100 years after the first reference in literature to acquired stuttering, the underlying physiopathological mechanisms remain unclear and merely speculative Orton, et al. and Travis, et al. firstly suggested a theory based on insufficient hemispheric dominance and subsequent "aberrant interhemispheric relationships" [8-10]. Later, it was proposed an explanation focused on damage to callosal path ways that can coordinate the activity of both hemispheres during speech, and may therefore explain the fact that lesions in either dominant or non-dominant hemisphere can cause stuttering [3]. Besides the former "inter-hemispheric" explanations, an "intra-hemispheric" mechanism postulates that stroke-related acquired stuttering results from dysfunction of neural processes involved in the cortex-basal ganglia-cortex network, and not from one specific lesion [11]. This may explain why infarcts in all lobes, cortical or subcortical, are associated with acquired stuttering. Additionally, recent data suggest that most adults have speech and language-related regions located in both hemispheres, and functional brain MRI studies demonstrated that many cortical and subcortical areas are activated while speaking; so even, in right-handed persons with typical left hemisphere dominance, language dysfunction could occur after cortical or subcortical right hemisphere damage, which is in concordance with our case [12-14].

In conclusion, we present a rare case of acquired stuttering after a cortical infarction in non-dominant hemisphere, and show that stuttering cannot be considered a focal neither lateralizing neurological sign, but rather it probably depends on the interaction between several cortical and subcortical structures. More research is needed to better understand the mechanisms underlying acquired stuttering after stroke.

\section{References}

1. Craig MC, Quaide A, Akram H, Zrinzo L, Tripoliti E (2014) A review of brain circuitries involved in stuttering. Front Hum Neurosci 17(8): 884.

2. Grant AC, Biousse V, Cook AA, Newman NJ (1999) Stroke-associated stuttering. Arch Neurol 56(5): 624627.
3. Soroker N, Bar-Israel Y, Schechier I, Solzi P (1990) Stuttering as a manifestation of right-hemispheric subcortical stroke. Eur Neurol 30(5): 268-269.

4. Helm NA, Butler RB, Benson DF (1978) Acquired stuttering. Neurology 28(11): 1159-1165.

5. Rosenbek J, Messert B, Collins M, Wertz RT (1978) Stuttering following brain damage. Brain Lang 6(1): 82-96.

6. Fleet WS, Heilman KM (1985) Acquired stuttering from a right hemisphere lesion in a right-hander. Neurology 35(9): 1343-1346.

7. Ardila A, Lopez MV (1986) Severe stuttering associated with right hemisphere lesion. Brain Lang 27(2): 239-246.

8. Lundgren K, Helm-Estabrooks N, Klein R (2010) Stuttering Following Acquired Brain Damage: A Review of the Literature. J Neurolinguistics 23(5): 447-454.

9. Orton ST (1928) A physiological theory of reading disability and stuttering in children. New Engl J Med 199: 1046-1052.

10. Travis LE (1978) The cerebral dominance theory of stuttering. J Speech Hear Disord 43(3): 278-281.

11. Theys C, De Nil L, Thijs V, van Wieringen A, Sunaert S (2013) A crucial role for the cortico-striato-cortical loop inthe pathogenesis of stroke-related neurogenic stuttering. Hum Brain Mapp 34(9): 2103-2112.

12. Dorsaint-Pierre R, Penhune VB, Watkins KE, Neelin $P$, Lerch JP, et al. (2006) Asymmetries of the planum temporale and Heschl's gyrus: Relationship to language lateralization. Brain 129(5): 1164-1176.

13. Margaret M Swanberg, Ziad S Nasreddine, Mario F Mendez JLC (2007) Textbook of Clinical Neurology. In: Textbook of Clinical Neurology $3^{\text {rd }}$ (Edn.), pp: 1392.

14. Fox PT (2003) Brain imaging in stuttering: Where next? J Fluency Disord 28(4): 265-272.

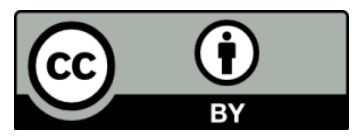

